\title{
Priorización para el análisis de información en salud pública. Red de conocimiento del Observatorio Nacional de Salud ${ }^{1}$
}

\author{
Prioritization for public health information analysis in Colombia. \\ Knowledge network at the National Health Observatory
}

\author{
Natalia Cediel-Becerra, Nelson Alvis-Guzmán, José Moreno-Montoya, \\ Gina A. Vargas-Sandoval y Carlos Castañeda-Orjuela
}

Recibido 15 enero 2016 / Enviado para modificación 4 octubre 2016 / Aceptado 16 enero 2017

\section{RESUMEN}

Objetivo Priorizar los grupos de enfermedades, población y métodos de análisis en salud pública según las preferencias de los actores de la red de conocimiento del Observatorio Nacional de Salud.

Método Se utilizó el método de Análisis Conjunto (AC), que consiste en la construcción de un modelo factorial completo tomando una muestra aleatoria de sujetos que deben, según un orden de importancia pre-establecido, identificar las preferencias de los atributos de determinado constructo. Los actores de la red de conocimiento que participaron fueron profesionales de universidades públicas, miembros de EPS, IPS, secretarias de salud departamental, organizaciones sin ánimo de lucro enfocadas en salud, centros de investigación especializados, de alcaldías, y corporaciones.

Resultados Los grupos de enfermedades prioritarios fueron: trastornos mentales y del comportamiento (13,8 \%), enfermedades cardiovasculares y circulatorias (13\%), neoplasmas $(10 \%)$ Diarrea, infecciones respiratorias, meningitis y otras enfermedades infecciosas comunes $(6,7 \%)$ y deficiencias nutricionales $(6,3 \%)$. Los criterios de priorización preferidos fueron la carga de enfermedad derivada y la dinámica epidemiológica del alto impacto. La información prioritaria de análisis fue la de determinantes sociales. El grupo poblacional de mayor preferencia fue la población general.

Conclusiones Se evidenció que los participantes perciben como prioritarias tanto las enfermedades crónicas como infecciosas concordante con la transición epidemiológica del país. La prioridad más sentida del sistema sanitario colombiano es la construcción de capacidad del recurso humano y el fortalecimiento del sistema de vigilancia en las regiones para la toma de decisiones en salud pública.

Palabras Clave: Agenda de prioridades en salud, salud pública, Colombia, métodos (fuente: DeCS, BIREME).

Objective To prioritize diseases, population and methods of analysis in public health according to the preferences of the stakeholders of the knowledge network of the National Health Observatory.

Method The Conjoint Analysis methodology (AC) was used; it consists on the construction of a complete factorial model taking a random sample of subjects that must identify the preferences of the attributes of a given construct according to a pre-established order of importance. The stakeholders of the knowledge network who participated were

1. Nota del Editor: El presente artículo es un producto del proyecto “Diseño e implementación de metodologías de información para el Observatorio Nacional de Salud-Instituto Nacional de Salud", realizado por la alianza estratégica Universidad Nacional de Colombia, Pontificia Universidad Javeriana, Universidad de los Andes, Fundación Santa Fe, Instituto CEIS y Fundación Salutia. Contrato RC-0053-2014, Instituto de Salud Pública, Facultad de Medicina, Universidad Nacional de Colombia y Departamento Administrativo de Ciencia, Tecnología e Innovación-Colciencias.
}

N.C.: MD. Veterinaria. Ph. D., M. Sc. Salud Pública. Universidad Nacional de Colombia, Facultad de Medicina. Bogotá, Colombia. nmcedielb@unal.edu.co

N.A.: MD. Ph. D. Economía de la Salud. Universidad de Cartagena. Colombia.

nalvis@yahoo.com

J.M.: Estadístico. Ph. D., M. Sc. Salud Pública. Universidad del Rosario, Bogotá, Colombia.

josemorenomontoya@gmail.com

G.V.: Enf. M. Sc. Epidemiología Clínica. Instituto Nacional de Salud-ONS. Bogotá, Colombia.galexandravs@gmail.com C.C.: MD. M. Sc. Economía. Instituto Nacional de Salud. Bogotá, Colombia. castanedao@ins.gov.co 
professionals from public universities, members of health promotion entities, health provision services, health departments, non-profit health organizations, specialized research centers, mayor's offices, and corporations.

Results The groups of priority diseases were mental and behavioral disorders (13.8\%), cardiovascular and circulatory diseases (13\%), neoplasms (10\%), diarrhea, respiratory infections, meningitis and other common infectious diseases $(6.7 \%)$, and nutritional deficiencies $(6.3 \%)$. The preferred prioritization criteria were the burden of disease and high-impact epidemiological dynamics. The analysis of priority information was the analysis of social determinants. The most preferred population was the general population.

Conclusions Participants perceive both chronic and infectious diseases as a priority, which is consistent with the epidemiological transition of the country. The priority for the Colombian health system is to strengthen the capacity of human resources and the surveillance system in different areas to have a better decision-making process in relation to public health.

Key Words: Health priority, methods, public health, Colombia (source: MeSH, NLM).

$\mathrm{L}$ a racionalidad económica en salud pública implica asignación eficiente de recursos. Así, la priorización como herramienta de planificación puede garantizar dicha racionalidad cuando es explícita y transparente, dado que minimiza la influencia de factores como el interés personal y las agendas políticas. Esto es más relevante en países en desarrollo en por la gran brecha entre la investigación en salud y el gasto sanitario. La priorización para la investigación y la información en salud pública, es necesaria para asignar fondos; promover la ciencia, la tecnología y la innovación en salud; fomentar el desarrollo de recurso humano para la investigación; negociar con organizaciones asociadas para obtener financiación y llevar a cabo iniciativas a largo plazo. El establecimiento de prioridades va de la mano del desarrollo (I-3). En ausencia de procesos de priorización, existe alto riesgo que la investigación en países de bajo y medio ingreso este determinada por donantes para sus propios propósitos o fallen en responder y explicar las necesidades de salud del país; por tanto, alinear las prioridades nacionales en investigación en salud con las políticas de los donantes puede distorsionar el rol de la investigación nacional en dichos países (4).

En Colombia, el Observatorio Nacional de Salud (ONs), de acuerdo con las funciones descritas en los Artículos 8 y $9^{\circ}$ de la Ley I 438 de 20 I I, debe generar evidencia para informar la toma de decisiones y guiar la formulación de políticas públicas en salud del país, con base en modelos de análisis que integren la información e identifiquen brechas de conocimiento requeridas para la formulación de proyectos de investigación.

El objetivo de este estudio fue conocer cuáles deben ser los eventos, la población a estudiar, los criterios más relevantes y los métodos analíticos prioritarios para el análisis de información en salud pública en Colombia.

\section{MÉTODOS}

El presente estudio aplicó el método de Conjoint Analysis (5) para priorizar eventos, población y métodos para el análisis de información en salud pública, entre febrero y junio de 2015 en Colombia.

\section{Atributos y niveles de la información}

Los participantes debieron elegir entre los 22 grupos de enfermedades que comprenden las ocho dimensiones del Plan Decenal en Salud Pública (PDSP) (6) y que dan cuenta del perfil epidemiológico potencial en el territorio nacional. Estos 22 grupos están descritos en el $2^{\circ}$ y zer Informe de Mortalidad Evitable del ons. Los grupos de enfermedades escogidos parten de la clasificación de grupos de causas del Estudio de Carga Global de Enfermedad 20Io. Los tres niveles usados en este estudio fueron:

- Criterios de priorización de enfermedades

- Grupos de población prioritarios

- Métodos de análisis prioritarios a realizar

Estos tres niveles se indagaron mediante tres preguntas:

Pregunta I: ¿Cuál es el criterio principal por el cual priorizó ese grupo de enfermedad? El primer nivel son los criterios para priorizar eventos en salud descritos por Krause y Balabanova en 20I I (7) (Cuadro I).

Cuadro 1. Criterios de priorización utilizados en la definición de atributos

\begin{tabular}{ll}
\hline \multicolumn{1}{c}{ Categorías } & \multicolumn{1}{c}{ Criterios de priorización } \\
\hline $\begin{array}{l}\text { Carga de } \\
\text { enfermedad. }{ }^{*}\end{array}$ & $\begin{array}{l}\text { - Severidencia } \\
\text { y escolar, tasas de hospitalización y } \\
\text { secuelas de la enfermedad } \\
\text { - Mortalidad / tasa de fatalidad }\end{array}$ \\
& - Potencial de emergencia \\
Dinámica & - Potencial de producir brotes \\
epidemiológica & - Tendencia en los últimos años \\
& - Evidencia de factores y grupos de riesgo \\
Necesidades de & - Atención pública incluyendo la agenda \\
información & política y la percepción de la comunidad \\
Oportunidad de & - Posibilidades de prevención \\
ganancia en salud & - Posibilidades de tratamiento \\
\hline
\end{tabular}

* En este estudio, la carga de enfermedad fue descrita como los años de vida ajustados por discapacidad -AVISA- que estiman la carga de la enfermedad, de acuerdo a las distintas causas o problemas de salud consideradas, en años vividos con discapacidad más los perdidos por muerte prematura.

Pregunta 2: ¿En cuál población particular es más relevante analizar ese grupo de enfermedad? En el segundo 
nivel, se toman los grupos de poblaciones prioritarios descritos en el PDSP (6) y se describen a continuación:

I. Niños, niñas y adolescentes

2. Adultez

3. Adulto mayor o en envejecimiento

4. Población en situación de discapacidad

5. Población víctima del conflicto armado

6. Grupos étnicos

7. Población con enfoque de género

8. Población ocupacionalmente expuesta

9. Toda la población/comunidad general

Pregunta 3: ¿Cuál método de análisis se debe utilizar para generar evidencia en ese grupo de enfermedad? El tercer nivel se toma de acuerdo con el tipo de método de análisis necesario para proporcionar mayor información sobre la situación en salud. El nivel de análisis según los posibles métodos analíticos utilizados por el ons en sus informes semestrales, son:

- Análisis de carga de enfermedad.

- Análisis de costos de enfermedad.

- Análisis de factores de riesgo.

- Análisis de determinantes sociales.

- Análisis de políticas públicas.

\section{Recolección de información}

Se diseñó y realizó prueba piloto, con los participantes del ons, de un cuestionario el cual posteriormente fue aplicado a los participantes en el estudio, mediante la plataforma monkey survey®.

\section{Plan de recolección de datos}

Por correo electrónico se invitaron a participar a actores claves de la Red de Conocimiento del ons. Se realizaron conversatorios presenciales en las cinco regiones del país con expertos y comunidad del sector sanitario y de la red de conocimientos del ons. Las ciudades escogidas fueron Cali, Bucaramanga, Leticia, Medellín, Cartagena y los actores participantes fueron: miembros de EPS e IPS, Universidades, Organizaciones sin ánimo de lucro enfocadas en salud, centros de investigación especializados, miembros de secretarias de salud departamental, alcaldías, sociedad civil, Parques Nacionales Naturales, ONG's en salud indígena.

\section{Análisis de la información}

El análisis conjunto (AC) busca la identificación de las preferencias en las respuestas en los niveles de atributos. De esta forma y a través de la identificación de los patrones de variación y co-variación de las respuestas, es posible estimar la importancia relativa de cada nivel de atributo $(8,9)$. En la práctica, el modelo se realiza mediante la toma de una muestra (aleatoria o de conveniencia justificada) de sujetos que deben, según un orden de importancia pre-establecido, identificar las ponderaciones de importancia de los atributos de determinado producto/constructo. A partir de esos niveles de atributo se calcula la importancia que cada atributo posee al momento de la elección de un determinado producto/ constructo. Simultáneamente, se conoce la utilidad parcial asociada a los diferentes niveles de atributo de los productos analizados. De esta forma, es posible conocer las configuraciones de los niveles de atributo que aumentan o disminuyen el atractivo de un determinado producto/constructo (IO).

Para el presente análisis se utilizó un modelo de preferencia de la utilidad global por lo que se usó un modelo factorial completo.

\section{Análisis estadístico}

A través de un modelo de mínimos cuadrados ordinarios (MCO) el modelo en función de los atributos y de los niveles, es el siguiente:

$$
y_{t}=\alpha+\sum_{i=1}^{I} \sum_{j=1}^{k_{i}} \beta_{i j} x_{i j}+e_{t}
$$

Donde:

$y_{t}$ : es el orden o la valoración de la preferencia sobre el estímulo $t$.

$\alpha$ : es el término constante (inobservado).

$\beta_{i j}$ : es la utilidad o partworth asociado al nivel $j$-ésimo

$\left(j=1,2, \ldots, k_{i}\right)$ del atributo i-ésimo $(i=1,2, \ldots, I)$.

$x_{i j}=1$ : si el nivel $j$-ésimo del atributo $i$-ésimo está presente en el estímulo $t$.

$x_{i j}=0$ : si el nivel $j$-ésimo del atributo $i$-ésimo no está presente en el estímulo $t$.

La base de la interpretación de resultados es el vector de utilidades o partworths $\beta$. Un valor alto o bajo de partworth significa que el nivel asociado proporciona al entrevistado una utilidad o preferencia alta o baja.

A partir de los partworths, el análisis conjunto también calcula la importancia relativa que los individuos atribuyen a los diferentes atributos que componen el producto/ constructo. Un atributo será más importante cuanto más grande sea la diferencia entre el partworth más elevado y el más bajo (en valores absolutos). Por tanto, se puede obtener la importancia de un atributo mediante:

$$
\operatorname{imp}_{i}=\left|\operatorname{máx}\left(\beta_{i j}\right)-\min \left(\beta_{i j}\right)\right| \forall i=1, \ldots, I \quad \forall j=1, \ldots, k_{i}
$$

Para poder comparar la importancia de cada factor se utiliza la importancia relativa:

$$
\operatorname{Pimp}_{i}=\frac{i m p_{i}}{\sum_{i=1}^{I} i m p_{i}} \cdot 100
$$


Todos los análisis se realizaron en el paquete estadístico STATA® en su versión I3.

\section{RESULTADOS}

\section{Características generales de los participantes en el ejercicio}

Se obtuvieron 222 respuestas de participantes, $58 \%$ mujeres. La mayoría del grupo $(6 \mathrm{I}, 0$ \%) con edades entre 26 y 40 años, la procedencia de los participantes fue de Amazonas ( I 2,6 \%), Antioquia ( I I,7 \%), Bogotá $(29,3 \%)$, Cartagena $(5,4 \%)$, Boyacá $(3,2 \%)$, Caldas $(0,9 \%)$, Caquetá $(0,9 \%)$, Cauca $(0,9 \%)$, Cesar $(0,9 \%)$, Chocó (I,4 \%), Córdoba (0,9\%), Cundinamarca $(7,7 \%)$, Huila (0,9\%), Magdalena (0,5\%), Meta (I,8\%), Norte de Santander (I,4 \%), Putumayo (0,9 \%), Quindío (0,5 \%), Santander (I2,6\%), Sucre $(0,9 \%)$, Tolima $(3,2 \%)$ y Valle $(\mathrm{I}, 8 \%)$. Las organizaciones a las que pertenecen eran Secretarias de Salud Departamental o Municipal (20,3\%), IPS y ESE $(8, \mathrm{I} \%)$, Asociaciones médicas, federaciones, corporaciones, ligas, fundaciones $(5,9 \%)$, Alcaldías (23\%), EPS (4,5\%), Universidades (I4,4\%), Sector publico central: Ministerio, INS, ONS, INVIMA, ICBF, Ecopetrol, Senado (I 5,3\%), Organismo internacional:
OPS, Naciones Unidas (0,9\%) y Sin respuesta $(7,2 \%)$. La mayoría tenía más de io años de experiencia (4I \%) y el 3 I $\%$ entre 6 y io.

\section{Respuestas de priorización a nivel nacional y departamental}

La proporción de respuestas según el grupo de enfermedad se muestran en la Tabla I. Se realizaron 240 observaciones a nivel nacional y 3II departamental. Los respondientes contestaron al menos una de las dos opciones de respuesta. Los trastornos mentales y de comportamiento, las enfermedades cardiovasculares y circulatorias, los neoplasmas, la diarrea, infecciones respiratorias, meningitis y otras enfermedades infecciosas comunes, y los trastornos maternos son los 5 grupos priorizados por los participantes a nivel nacional. A nivel departamental, los resultados fueron similares, incorporando las deficiencias nutricionales.

En la Tabla 2 se describen las respuestas según las preguntas de la matriz de priorización. A nivel nacional las respuestas más frecuentes fueron: para la Pregunta I, Carga de Enfermedad (39\%); para la Pregunta 2, Población en general (48\%) y, para la Pregunta 3, análisis de determinantes sociales $(27,3 \%)$. A nivel departamental

Tabla 1. Enfermedades más escogidas a nivel nacional y departamental

\begin{tabular}{|c|c|c|c|c|}
\hline \multirow{2}{*}{ Grupo de enfermedades } & \multicolumn{2}{|c|}{ Nacional } & \multicolumn{2}{|c|}{ Departamental } \\
\hline & Frecuencia & $\%$ & Frecuencia & $\%$ \\
\hline 1. VIH/SIDA y Tuberculosis. & 12 & 5,0 & 16 & 5,1 \\
\hline $\begin{array}{l}\text { 2. Diarrea, infecciones respiratorias, meningitis y otras } \\
\text { enfermedades infecciosas comunes. }\end{array}$ & 16 & 6,7 & 28 & 9,0 \\
\hline 3. Enfermedades desatendidas y malaria & 14 & 5,8 & 21 & 6,7 \\
\hline 4. Trastornos maternos & 16 & 6,6 & 18 & 5,7 \\
\hline 5. Trastornos neonatales & 3 & 1,2 & 8 & 2,5 \\
\hline 6. Deficiencias nutricionales & 15 & 6,2 & 22 & 7,0 \\
\hline 7. Otros enfermedades transmisibles, maternas, neonatales & 4 & 1,6 & 5 & 1,6 \\
\hline 8. Neoplasmas & 24 & 10,0 & 24 & 7,7 \\
\hline 9- Enfermedades cardiovasculares y circulatorias & 31 & 12,9 & 43 & 13,8 \\
\hline 10. Enfermedades respiratorias crónicas & 4 & 1,6 & 10 & 3,2 \\
\hline 11. Cirrosis hepática & 1 & 0,4 & 1 & 0,3 \\
\hline 12. Enfermedades digestivas - excepto cirrosis-. & 4 & 1,6 & 6 & 1,9 \\
\hline 13. Trastornos neurológicos & 6 & 2,5 & 2 & 0,6 \\
\hline 14. Trastornos mentales y de comportamiento & 33 & 13,7 & 46 & 14,9 \\
\hline 15. Diabetes y enfermedades urogenitales, de sangre y endocrinas & 8 & 3,3 & 12 & 3,8 \\
\hline 16. Trastornos musculo-esqueléticos & 5 & 2,1 & 2 & 0,6 \\
\hline 17. Otras enfermedades no transmisibles & 1 & 0,4 & 2 & 0,6 \\
\hline 18. Lesiones de transporte & 7 & 2,9 & 3 & 0,9 \\
\hline 19. Lesiones no intencionales diferentes a lesiones de transporte & 2 & 0,8 & 3 & 0,9 \\
\hline 20. Lesiones auto infligidas y agresiones & 8 & 3,3 & 8 & 2,5 \\
\hline $\begin{array}{l}\text { 21. Enfermedades o lesiones debido a fuerzas de la naturaleza, } \\
\text { guerra - violencia }\end{array}$ & 12 & 5,0 & 9 & 2,8 \\
\hline $\begin{array}{l}\text { 22. Enfermedades producidas por riesgos laborales y } \\
\text { ocupacionales }\end{array}$ & 11 & 4,5 & 18 & 5,7 \\
\hline 23. Otra enfermedad o grupo de enfermedad (especifique) & 3 & 1,2 & 4 & 1,2 \\
\hline Total & 240 & 100,0 & 311 & 100,0 \\
\hline
\end{tabular}


Tabla 2. Respuestas a las tres preguntas de la matriz de priorización a nivel nacional

\begin{tabular}{|c|c|c|c|c|}
\hline \multirow{2}{*}{ Grupo de enfermedades } & \multicolumn{2}{|c|}{ Nacional } & \multicolumn{2}{|c|}{ Departamental } \\
\hline & Frecuencia & $\%$ & Frecuencia & $\%$ \\
\hline \multicolumn{5}{|c|}{ Pregunta 1 ¿Cuál es el criterio principal por el cual priorizó ese grupo de enfermedad? } \\
\hline 1. Carga de enfermedad significativa & 93 & 39,0 & 124 & 40,4 \\
\hline 2. Dinámica epidemiológica de alto impacto & 51 & 21,4 & 79 & 25,7 \\
\hline 3. Gran necesidad de información & 46 & 19,3 & 50 & 16,2 \\
\hline 4. Agenda política y alta percepción de la comunidad & 25 & 10,5 & 16 & 5,2 \\
\hline 5. Alta oportunidad de ganancia en salud & 22 & 9,2 & 38 & 12,3 \\
\hline 6. Otro & 1 & 0.4 & & \\
\hline \multicolumn{5}{|c|}{ Pregunta 2 ¿En cuál población particular es más relevante analizar ese grupo de enfermedad? } \\
\hline 1. Niños, niñas y adolescentes - & 41 & 17,2 & 69 & 22,4 \\
\hline 2. Adultez - & 39 & 16,3 & 38 & 12,3 \\
\hline 3. Adulto mayor o en envejecimiento - & 11 & 4,6 & 23 & 7,4 \\
\hline 4. Población en situación de discapacidad - & 1 & 0,4 & 1 & 0,3 \\
\hline 5. Población víctima del conflicto armado - & 7 & 2,9 & 4 & 1,3 \\
\hline 6. Grupos étnicos - & 5 & 2,1 & 9 & 2,9 \\
\hline 7. Población con enfoque de género - & 12 & 5,0 & 11 & 3,5 \\
\hline 8. Población ocupacionalmente expuesta - & 7 & 2,9 & 15 & 4,8 \\
\hline 9. Toda la población/comunidad general & 115 & 48,3 & 139 & 44,6 \\
\hline \multicolumn{5}{|c|}{ Pregunta 3 ¿Cuál método de análisis se debe utilizar para generar evidencia en ese grupo de enfermedad? } \\
\hline 1. Análisis de carga de enfermedad & 44 & 18,4 & 69 & 22,4 \\
\hline 2. Análisis de costos de enfermedad & 39 & 16,3 & 38 & 12,3 \\
\hline 3. Análisis de factores de riesgo & 54 & 22,6 & 73 & 23,7 \\
\hline 4. Análisis de determinantes sociales. & 65 & 27,3 & 92 & 29,9 \\
\hline 5. Análisis de políticas públicas & 36 & 15,1 & 35 & 11,4 \\
\hline Total & 238 & & 307 & \\
\hline
\end{tabular}

las respuestas fueron similares: Carga de enfermedad $(40,3 \%)$, población en general (44.63\%) y análisis de determinantes sociales $(29,9 \%)$ seguido de análisis de factores de riesgo $(23 \%)$.

\section{Modelos multinivel de regresión logística ordinal para las respuestas a nivel Nacional y departamental}

\section{Nacional}

Luego del análisis estadístico, influyen en la decisión únicamente la edad del respondiente y la opción de preguntas 2, a decir, la población en la cual priorizar. El modelo ajustado indicó, una vez tomado en cuenta el efecto de la agrupación de las respuestas y debido al hecho de que cada respondiente seleccionó dos opciones de respuesta, que las características preponderantes en cuanto a la definición de los grupos de enfermedades prioritarios son aquellas que dependen del grupo poblacional asociado con un Coeficiente $=0,05$ I 9 IC $95 \%$ $(0,0065-0,0973)$.

Hubo I I $5(48,3 \%)$ observaciones que indican preferencia de los respondientes por indagar y priorizar las enfermedades consultadas en toda la población; siendo este el criterio más importante para priorizar las enfermedades que seleccionaron los participantes, seguido por el grupo de niños y niñas 4I $(I 7,2 \%)$.
Se llevó a cabo una prueba convencional de medias con el ánimo de verificar en el caso nacional, dentro de los grupos seleccionados de preguntas (b: grupo poblacional) cuales opciones de respuesta mostraban diferencias significativas respecto a las demás. Los resultados indican que de las preguntas de grupo poblacional, cuya variación implicó cambios en la elección del grupo de enfermedades los ítems: Adultez ( $\mathrm{p}<\mathrm{O}, \mathrm{OOI}$ ), adulto mayor o en envejecimiento ( $\mathrm{p}=0, \mathrm{O} I 8)$, población en discapacidad $(\mathrm{p}<\mathrm{0}, \mathrm{OOI})$, población víctima del conflicto armado $(\mathrm{p}<\mathrm{0}, \mathrm{OOI})$, población ocupacionalmente expuesta $(\mathrm{p}=0,004)$ y toda la población $(\mathrm{p}<\mathrm{O}, \mathrm{OOI})$ variaron significativamente. Esto significa que tales proporciones se comportaron de forma distinta entre ellas y los grupos de enfermedades, verificando poco acuerdo en la elección de las enfermedades prioritarias vistas a nivel nacional desde el enfoque de los grupos poblacionales.

\section{Departamental}

Influyen en la decisión únicamente la edad del respondiente y la opción de pregunta I, es decir los criterios de priorización. El modelo ajustado indica que las características preponderantes para la definición de grupos de enfermedades prioritarios son aquellas que dependen de los criterios de priorización, es decir: carga de enfermedad significativa, dinámica epidemiológica de alto impac- 
to, gran necesidad de información, agenda política y alta percepción de la comunidad y alta oportunidad de ganancia en salud. Se observó una variabilidad significativa en cuanto a la edad de los entrevistados, razón por la que ésta es considerada significativa en el modelo, como se presenta en la Tabla 3 .

Tabla 3. Variables y coeficientes

\begin{tabular}{ccc}
\hline $\begin{array}{c}\text { Variables asociadas } \\
\text { con la elección }\end{array}$ & Coeficiente & IC 95\% \\
\hline $\begin{array}{c}\text { Preguntas grupo criterios } \\
\text { de priorización }\end{array}$ & 0,0802999 & $(0,0107-0,1498)$ \\
\hline Edad del respondiente & 0,1835776 & $(0,074-0,2924)$ \\
\hline
\end{tabular}

Las respuestas por grupo de enfermedad correspondientes a la pregunta I, que resultó ser la más influyente en la decisión de por qué priorizar las enfermedades seleccionadas se pueden resumir de la siguiente manera: Hubo I $24(40,39 \%)$ de las respuestas que indican preferencia por el aspecto de carga de enfermedad; siendo este el criterio más importante para priorizar las enfermedades que seleccionaron los participantes. Siguió la dinámica epidemiológica de alto impacto con $79(25,7 \%)$ elecciones y 50 respuestas para necesidad de información (I6,2 \%). El criterio menos citado como relevante para escoger un grupo de enfermedad prioritario fue el de Agenda política y alta percepción de la comunidad con I 6 coincidencias.

Desde la perspectiva de las preguntas asociadas con impacto los grupos de enfermedades se comportan como se muestra a continuación: trastornos mentales y de comportamiento, enfermedades cardiovasculares y circulatorias, diarrea, infecciones respiratorias, meningitis y otras enfermedades infecciosas comunes, neoplasmas, enfermedades desatendidas y malaria, deficiencias nutricionales. En conjunto estas seis opciones consolidan 59, I \% del total de elecciones.

Se llevó a cabo una prueba de medias para verificar, en el caso departamental, dentro de los grupos seleccionados de preguntas (a: de impacto) cuales opciones de respuesta mostraban diferencias significativas respecto a las demás. Los resultados indican que de las preguntas de impacto (solo las del grupo a), cuya variación implicó cambios en la elección del grupo de enfermedades, el ítem 3 (necesidad de información) es significativamente diferente en su comportamiento respecto a las demás preguntas ( $\mathrm{p}=$ o.o I 8). Esto significa que la opción tres se comportó diferencialmente de las demás opciones de respuesta dentro del grupo de preguntas de impacto.

\section{DISCUSIÓN}

Los resultados en este estudio muestran que del listado de 22 grupos de enfermedades, las enfermedades cardio- vasculares (ECV) y a los trastornos mentales y de comportamiento fueron seleccionados como prioridades para el análisis de información en salud pública en Colombia. Probablemente las razones que explican estos resultados incluyen la transición epidemiológica, el crecimiento poblacional y el envejecimiento de la población (I I).

Por otra parte, la carga de enfermedad fue el criterio más importante para priorizar enfermedades y las enfermedades que obtuvieron los puntajes más altos en este estudio, fueron las mismas enfermedades que Murray y colaboradores identificaron midiendo carga de enfermedad a nivel mundial ( I I ). De acuerdo con el Quinto Informe del ONS, sobre carga de enfermedad de enfermedades crónicas no transmisibles, se evidencia que en Colombia, las ECV son el grupo de enfermedades que generan mayor carga de enfermedad en el mundo, ocupando el primer lugar en cuanto a mortalidad y morbilidad, causando el 3I \% de todas las muertes en el planeta, con I7,5 millones de muertes (I2). En este estudio, las ECV ocuparon el segundo lugar de prioridades y la razón principal por la cual escogieron ese grupo fue por la carga de enfermedad.

Las enfermedades transmisibles, maternas, las enfermedades inmunoprevenibles (diarrea, infecciones respiratorias, meningitis y otras enfermedades infecciosas comunes) y las enfermedades nutricionales estuvieron dentro de los grupos más escogidos como prioritarios a nivel departamental y nacional. Lo anterior demuestra la heterogeneidad de prioridades en salud en el perfil epidemiológico colombiano y el fenómeno de transición epidemiológica representa un reto para la priorización de acciones de prevención y control y en general para la toma de decisiones por parte de las autoridades sanitarias. Frente a esto, los hallazgos del 2do Informe del oNs (I3) evidenciaron que en Colombia las enfermedades no transmisibles y lesiones son las principales causas de muerte en los I4 años de análisis y aunque la mortalidad infantil y la mortalidad en menores de cinco años han registrado una disminución del 37,5\% y 39,0 \%, respectivamente, se observaron desigualdades por departamentos.

Además de identificar grupos de enfermedad prioritarios, este estudio identificó los análisis de información y los grupos poblacionales más relevantes para orientar la política pública sanitaria. Los análisis de información prioritarios fueron los análisis de determinantes sociales y los análisis de factores de riesgo a nivel departamental y nacional. El grupo poblacional más escogido fue la población general seguido de la población de niños, niñas y adolescentes. Frente a estos resultados, resulta interesante ver que la población prefirió estudios que ya están desarrollándose por el ons, y que hicieron parte del tercer Informe en 20I3, como ejemplo los estudios multinivel 
para mortalidad materna e infantil. Los resultados más relevantes de dicho informe evidencian que la mortalidad infantil se asoció significativamente con características individuales como: el sexo del niño, la educación de la madre; el número de semanas gestacionales al nacimiento; los meses de lactancia materna; la pertenencia a algún grupo étnico; el número de miembros en el hogar, la pobreza en el hogar y características del entorno como la pobreza multidimensional municipal y departamental (I4).

Desde el punto de vista metodológico, la estrategia buscó identificar, en términos de frecuencia y variación, los patrones de respuesta más comunes en la población. En principio lo que el modelo busca es consolidar un sistema de "puntuación" que incluya simultáneamente (y tome en cuenta su variación y co-variacíon) aspectos de los respondientes y de las enfermedades a evaluar; de este modo, el método no valora el tipo y orden de los criterios sino la ponderación (valor) que se les ha dado entre los diferentes grupos de respondientes. En términos de frecuencia, el patrón de respuestas más común para un grupo de enfermedades, el tipo de impacto y de análisis requerido "co-variaron" de forma que reflejó asociaciones. A diferencia de un análisis de frecuencias simples o compuestas, la modelación permite la valoración simultánea de combinar la variación debida a los grupos de preguntas, tipos de respondientes, conglomeración de las respuestas por respondiente y tipo de impacto de las enfermedades en cuestión. En ambos casos, departamental y nacional, los resultados muestran que existe un aceptable grado de evidencia estadística respecto al comportamiento de las elecciones hechas por los entrevistados. Se dio acuerdo en la elección de las enfermedades prioritarias.

Con el uso del método de análisis conjunto, se pretendió superar una de las limitantes de los ejercicios de priorización basados en opiniones de expertos, ya que debido a la alta especialización y el nivel de conocimiento a profundidad de algunos profesionales, se pierde la noción de contexto de las problemáticas en salud de poblaciones a nivel local (5).

Por otro lado, al incluir 22 territorios de diferentes regiones del país, se buscó incrementar la representatividad de los participantes y por ende la generalización de los resultados a nivel nacional, aunque es bien claro que las prioridades departamentales no necesariamente se corresponde con las nacionales. Sin embargo, una de las limitantes del presente estudio, es la gran participación de cuatro departamentos (Santander, Amazonas, Bogotá y Antioquia) y la poca o ninguna participación de los demás, limitando el alcance de las conclusiones en los grupos de enfermedad prioritarios solo a los departamentos participantes. Otra limitante de este estudio es que el grupo de enfermedades incluidos en el infor- me de carga mundial de la enfermedad publicada en el informe del 20I0, no es exhaustiva de las condiciones que se presentan en los países en desarrollo y esto puede haber generado que problemas de salud pública, no seas considerado como prioritario en nuestro medio por no haber sido incluidos. Este potencial problema se intentó resolver, mediante la inclusión en la encuesta de una opción de pregunta nombrada como "otro", en donde los participantes nombraron las siguientes: Intoxicación por metales pesados por minería, enfermedades emergentes, infecciosas zoonóticas y resistencia antimicrobiana.

Este es el primer ejercicio de priorización para el análisis de información en salud pública desarrollado en Colombia y el primer ejercicio utilizando el método de análisis conjunto para fines de establecimiento de prioridades en salud pública. Estos resultados ilustran que el análisis conjunto, puede ser usado como una herramienta potencial para priorizar temas, eventos, problemas, o condiciones en salud pública, superando las siguientes limitaciones: la ponderación y asignación de puntajes subjetivos a los criterios de enfermedad por parte de los expertos, la incorrecta suposición de independencia entre los criterios de enfermedad, los sesgos asociados con los nombres de las enfermedades y la limitada participación de expertos (I 5).

Aunque no existe un modelo metodológico aceptado como "gold estándar" para el establecimiento de prioridades a nivel nacional o internacional, si hay aspectos comunes de buenas prácticas para el establecimiento de prioridades en investigación en salud (I6). En este sentido, este estudio hace parte del estudio piloto para el diseño de la guía metodológica de priorización del ons en el cual se integraron y adaptaron los tres abordajes comprehensivos descritos por la matriz de enfoque combinada $3 \mathrm{D}$ desarrollada por el Foro Global (I7), el enfoque de seis pasos del Consejo de Investigación en Salud para el Desarrollo (en inglés COHRED) ( I 8) y el enfoque de 9 temas buenas prácticas para el establecimiento de prioridades en investigación en salud desarrollado por la oms (2)

Agradecimientos: Los autores desean agradecer a las personas e instituciones por su valioso apoyo y colaboración en el diseño, desarrollo, mejoramiento y publicación de este proyecto: Equipo de trabajo del Instituto de Salud Pública - Universidad Nacional de Colombia; Observatorio de Salud Pública de Santander; Laboratorio de Salud Pública del Amazonas; Grupo de Investigación Centauro - Universidad de Antioquia; Escuela de Salud Pública - Universidad del Valle: Universidad de Cartagena; EPS Mutual Ser; Dr. Juan Pablo Albanés; Dra. Luz Mila Murcia; Dr. Andrés Londoño; Miembros del ons.

Conflictos de interés: Ninguno. 


\section{REFERENCIAS}

1. Gilsdorf A, Krause G. Prioritisation of infectious diseases in public health: feedback on the prioritisation methodology, 15 July 2008 to 15 January 2009; 2011.

2. Viergever RF, Olifson S, Ghaffar A, Terry RF. A checklist for health research priority setting: nine common themes of good practice. Health research policy and systems/BioMed Central. 2010;8:36.

3. World Health Organization. Setting priorities in communicable disease surveillance. WHO/CDS/EPR/LYO/20063; 2006.

4. Ali N, Hill C, Kennedy A, IJsselmuiden C. What factors influence national health research agendas in low and middle income countries. Perspectives of health research from six countries. 2006;11.

5. Bridges JF, Hauber AB, Marshall D, Lloyd A, Prosser LA, Regier DA, et al. Conjoint analysis applications in health--a checklist: a report of the ISPOR Good Research Practices for Conjoint Analysis Task Force. Value in health : the journal of the International Society for Pharmacoeconomics and Outcomes Research. 2011;14(4):403-13.

6. Ministerio de Salud y Protección Social. Plan Decenal de Salud Pública PDSP, 2012-2012. La salud en Colombia la construyes tú, . Bogotá D.C. Colombia 2013 [cited 2015. Available from: https://goo.gl/6yiDrj.

7. Krause G. How can infectious diseases be prioritized in public health? EMBO reports. 2008;9(S1):S22-S7.

8. Haley RI. Benefit segmentation: a decision-oriented research tool. The Journal of Marketing. 1968;30-5.

9. Wind $Y$. Issues and advances in segmentation research. Journal of marketing research. 1978;317-37.

10. Lévy J-P, Varela J. Análisis multivariable para las ciencias sociales. Madrid, Editorial Pearson Educación; 2003.
11. Murray CJ, Vos T, Lozano R, Naghavi M, Flaxman AD, Michaud C, et al. Disability-adjusted life years (DALYs) for 291 diseases and injuries in 21 regions, 1990-2010: a systematic analysis for the Global Burden of Disease Study 2010. Lancet (London, England). 2012;380(9859):2197-223.

12. Instituto Nacional de Salud, Observatorio Nacional de Salud. Quinto Informe ONS: carga de enfermedad por enfermedades crónicas no transmisibles y discapacidad en Colombia. . Imprenta Nacional de Colombia, Bogotá, DC.; 2015.

13. Instituto Nacional de Salud, Observatorio Nacional de Salud. Segundo Informe ONS: Mortalidad 1998-2011 y situación de salud en los municipios de frontera terrestre en Colombia. Imprenta Nacional de Colombia, Bogotá, DC, Colombia; 2013.

14. Instituto Nacional de Salud, Observatorio Nacional de Salud. Tercer Informe ONS: Mortalidad evitable en Colombia para 1998-2011. Imprenta Nacional de Colombia, Bogotá, DC, Colombia; 2014.

15. Ng V, Sargeant JM. A quantitative approach to the prioritization of zoonotic diseases in North America: a health professionals' perspective. PloS one. 2013;8(8):e72172.

16. McGregor S, Henderson KJ, Kaldor JM. How are health research priorities set in low and middle income countries? A systematic review of published reports. PloS one. 2014;9(9):e108787.

17. Global Forum for Health Research. The 3D Combined Approach Matrix: An improved tool for settinng priorities in research for health 2009 [cited 2015]. Available from: https:// goo.gl/5Fq3sU.

18. Montorzi G, De Haan S, IJsselmuiden C. Council on Health Research for Development (COHRED). Priority Setting for Research for Health: A Management Process for Countries; 2010. 